\title{
AN ANALOGUE OF COWLING-PRICE'S THEOREM FOR THE Q-FOURIER-DUNKL TRANSFORM
}

\author{
Azzedine Achak, Radouan Daheh, Najat Safouane and El Mehdi Loualid
}

\author{
(C) 2020 by University of Niš, Serbia | Creative Commons License: CC BY-NC-ND
}

\begin{abstract}
The Q-Fourier-Dunkl transform satisfies some uncertainty principles in a similar way to the Euclidean Fourier transform. By using the heat kernel associated to the Q-Fourier-Dunkl operator, we have established an analogue of Cowling-Price, Miyachi and Morgan theorems on $\mathbb{R}$ by using the heat kernel associated to the QFourier-Dunkl transform.

Keywords: Cowling-Price's theorem; Miyachi's theorem; Uncertainty Principles; QFourier-Dunkl transform.
\end{abstract}

\section{Introduction}

There are many theorems which state that a function and its classical Fourier transform on $\mathbb{R}$ cannot simultaneously be very small at infinity. This principle has several versions which were proved by M.G. Cowling and J.F. Price [3] and Miyachi [6]. In this paper, we will study an analogue of Cowling-Price's theorem and Miyachi's theorem for the Q-Fourier-Dunkl transform. Many authors have established the analogous of Cowling-Price's theorem in other various setting of harmonic analysis (see for instance [5]) The outline of the content of this paper is as follows.

Section 2 is dedicated to some properties and results concerning the Q-FourierDunkl transform. In Section 3 we give an analogue of Cowling-Price's theorem, Miyachi's theorem, and Morgan's theorem for the Q-Fourier-Dunkl transform. Let us now be more precise and describe our results. To do so, we need to introduce some notations. Throughout this paper $\alpha>\frac{-1}{2}$. Notice that if $\alpha=\frac{-1}{2}$ then the space is the classical Lebesgue one, we can follow in this case the procedures for similar transforms, such as the Fourier transform (see for example $[3,6]$ ).

$$
Q(x)=\exp \left(-\int_{0}^{x} q(t) d t\right), \quad x \in \mathbb{R}
$$

Received January 06, 2018; accepted March 18, 2019

2010 Mathematics Subject Classification. Primary 42A38; Secondary 44A35, 34B30 
where $q$ is a $\mathcal{C}^{\infty}$ real-valued odd function on $\mathbb{R}$.

- $L_{\alpha}^{p}(\mathbb{R})$ the class of measurable functions $f$ on $\mathbb{R}$ for which $\|f\|_{p, \alpha}<\infty$, where

$$
\|f\|_{p, \alpha}=\left(\int_{\mathbb{R}}|f(x)|^{p}|x|^{2 \alpha+1} d x\right)^{\frac{1}{p}}, \quad \text { if } 1<p<\infty
$$

and $\|f\|_{\infty, \alpha}=\|f\|_{\infty}=\operatorname{esssup}_{x \in \mathbb{R}}|f(x)|$.

- $L_{Q}^{p}(\mathbb{R})$ the class of measurable functions $f$ on $\mathbb{R}$ for which $\|f\|_{p, Q}=\|Q f\|_{p, \alpha}<$ $\infty$, where $Q$ is given by (1.1).

We consider the first singular differential-difference operator $\Lambda$ defined on $\mathbb{R}$

$$
\Lambda f(x)=f^{\prime}(x)+\left(\alpha+\frac{1}{2}\right) \frac{f(x)-f(-x)}{x}+q(x) f(x)
$$

where $q$ is a $\mathcal{C}^{\infty}$ real-valued odd function on $\mathbb{R}$. For $q=0$ we regain the Dunkl operator $\Lambda_{\alpha}$ associated with reflection group $\mathbb{Z}_{2}$ on $\mathbb{R}$ given by

$$
\Lambda_{\alpha} f(x)=f^{\prime}(x)+\left(\alpha+\frac{1}{2}\right) \frac{f(x)-f(-x)}{x} .
$$

\subsection{Q-Fourier-Dunkl Transform}

The following statements are proved in [1]

Lemma 1.1. 1. For each $\lambda \in \mathbb{C}$, the differential-difference equation

$$
\Lambda u=i \lambda u, \quad u(0)=1
$$

admits a unique $\mathcal{C}^{\infty}$ solution on $\mathbb{R}$, denoted by $\Psi_{\lambda}$, given by

$$
\Psi_{\lambda}(x)=Q(x) e_{\alpha}(i \lambda x)
$$

where $e_{\alpha}$ denotes the one-dimensional Dunkl kernel defined by

$$
e_{\alpha}(z)=j_{\alpha}(i z)+\frac{z}{2(\alpha+1)} j_{\alpha+1}(z) \quad(z \in \mathbb{C}),
$$

and $j_{\alpha}$ being the normalized spherical Bessel function of index $\alpha$ given by

$$
j_{\alpha}(z)=\Gamma(\alpha+1) \sum_{n=0}^{\infty} \frac{(-1)^{n}\left(\frac{z}{2}\right)^{2 n}}{n ! \Gamma(n+\alpha+1)} \quad(z \in \mathbb{C}) .
$$

2. For all $x \in \mathbb{R}, \lambda \in \mathbb{C}$ and $n=0,1, \ldots$ we have

$$
\left|\frac{\partial^{n}}{\partial \lambda^{n}} \Psi_{\lambda}(x)\right| \leq Q(x)|x|^{n} e^{|\operatorname{Im} \lambda||x|} .
$$

In particular

$$
\left|\Psi_{\lambda}(x)\right| \leq Q(x) e^{|I m \lambda||x|}
$$


3. For all $x \in \mathbb{R}, \lambda \in \mathbb{C}$, we have the Laplace type integral representation

$$
\Psi_{\lambda}(x)=a_{\alpha} Q(x) \int_{-1}^{1}\left(1-t^{2}\right)^{\alpha-\frac{1}{2}}(1+t) e^{i \lambda x t} d t
$$

where $a_{\alpha}=\frac{2 \Gamma(\alpha+1)}{\sqrt{\pi} \Gamma\left(\alpha+\frac{1}{2}\right)}$.

Definition 1.1. The Q-Fourier-Dunkl transform associated with $\Lambda$ for a function in $L_{Q}^{1}(\mathbb{R})$ is defined by

$$
\mathcal{F}_{Q}(f)(\lambda)=\int_{\mathbb{R}} f(x) \Psi_{-\lambda}(x) x^{2 \alpha+1} d x .
$$

Theorem 1.1. $\quad$ 1. Let $f \in L_{Q}^{1}(\mathbb{R})$ such that $\mathcal{F}_{Q}(f) \in L_{\alpha}^{1}(\mathbb{R})$. Then for allmost $x \in \mathbb{R}$ we have the inversion formula

$$
f(x)(Q(x))^{2}=m_{\alpha} \int_{\mathbb{R}} \mathcal{F}_{Q}(f)(\lambda) \Psi_{\lambda}(x)|\lambda|^{2 \alpha+1} d \lambda,
$$

where

$$
m_{\alpha}=\frac{1}{2^{2(\alpha+1)}(\Gamma(\alpha+1))^{2}} .
$$

2. For every $f \in L_{Q}^{2}(\mathbb{R})$, we have the Plancherel formula

$$
\int_{\mathbb{R}}|f(x)|^{2}(Q(x))^{2}|x|^{2 \alpha+1} d x=m_{\alpha} \int_{\mathbb{R}}\left|\mathcal{F}_{Q}(f)(\lambda)\right|^{2}|\lambda|^{2 \alpha+1} d \lambda .
$$

3. The Q-Fourier-Dunkl transform $\mathcal{F}_{Q}$ extends uniquely to an isometric isomorphism from $L_{Q}^{2}(\mathbb{R})$ onto $L_{\alpha}^{2}(\mathbb{R})$.

The heat kernel $N(x, s), x \in \mathbb{R}, s>0$, associated with the Q-Fourier-Dunkl transform is given by

$$
N(x, s)=m_{\alpha} \frac{e^{-\frac{x^{2}}{4 s}}}{(2 s)^{\alpha+\frac{1}{2}} Q(x)} .
$$

Some basic properties of $N(x, s)$ are the following:

$$
\text { - } N(x, s) Q^{2}(x)=m_{\alpha} \int_{\mathbb{R}} e^{-s y^{2}} \Psi_{y}(x)|y|^{2 \alpha+1} d y \text {. }
$$

- $\mathcal{F}_{Q}(N(., s))(x)=e^{-s x^{2}}$.

we define the heat functions $W_{l}, l \in \mathbb{N}$ as

$$
Q^{2}(x) W_{l}(x, s)=\int_{\mathbb{R}} y^{l} e^{-\frac{y^{2}}{4 s}} \Psi_{y}(x)|y|^{2 \alpha+1} d y
$$




$$
\mathcal{F}_{Q}\left(W_{l}(., s)\right)=i^{l} y^{l} e^{-s y^{2}}
$$

The intertwining operators associated with a Q-Fourier-Dunkl transform on the real line is given by

$$
X_{Q}(f)(x)=a_{\alpha} Q(x) \int_{-1}^{1} f(t x)\left(1-t^{2}\right)^{\alpha-\frac{1}{2}} d t
$$

its dual is given by

$$
{ }^{t} X_{Q}(f)(y) a=\quad a_{\alpha} \int_{|x| \geq|y|} f(x) Q(x) \operatorname{sgn}(x)\left(x^{2}-y^{2}\right)^{\alpha-\frac{1}{2}}(x+y) d x
$$

${ }^{t} X_{Q}$ can be written as

$$
{ }^{t} X_{Q}(f)(y)=a_{\alpha} \int_{\mathbb{R}} f(x) Q(x) d \nu_{y}(x),
$$

where

$$
d \nu_{y}(x)=a_{\alpha} \chi_{\{|x| \geq|y|\}} \operatorname{sgn}(x)\left(x^{2}-y^{2}\right)^{\alpha-\frac{1}{2}}(x+y) d x
$$

and $\chi_{\{|x| \geq|y|\}}$ denote the characteristic function with support in the set $\{x \in$ $\mathbb{R} /|x| \geq|y|\}$.

Proposition 1.1. If $f \in L_{Q}^{1}(\mathbb{R})$ then ${ }^{t} X_{Q}(f) \in L^{1}(\mathbb{R})$ and $\left\|{ }^{t} X_{Q}(f)\right\|_{1} \leq\|f\|_{1, Q}$.

For every $f \in L_{Q}^{1}(\mathbb{R})$

$$
\mathcal{F}_{Q}=\mathcal{F}^{t} X_{Q}(f),
$$

where $\mathcal{F}$ is the usual Fourier transform defined by

$$
\mathcal{F}(f)(\lambda)=\int_{\mathbb{R}} f(x) e^{-i \lambda x} d x .
$$

\section{Cowling-Price's Theorem for the Q-Fourier-Dunkl Transform}

Theorem 2.1. Let $f$ be a measurable function on $\mathbb{R}$ such that

$$
\int_{\mathbb{R}} \frac{e^{a p x^{2}} Q^{p}(x)|f(x)|^{p}}{(1+|x|)^{k}}|x|^{2 \alpha+1} d x<\infty
$$

and

$$
\int_{\mathbb{R}} \frac{e^{b q \xi^{2}}\left|\mathcal{F}_{Q}(f)(\xi)\right|^{q}}{(1+|\xi|)^{m}} d \xi<\infty
$$

for some constants $a, b>0, k>0, m>1$ and $1 \leq p, r \leq+\infty$.

i) If $a b>\frac{1}{4}$, then $f=0$ almost everywhere. 
ii) If $a b=\frac{1}{4}$, then $f(x)=P(x) N(x, b)$ where $P$ is a polynomial with $\operatorname{deg} P \leq \min \left\{\frac{k}{p}+\frac{2 \alpha+1}{p^{\prime}}, \frac{m-1}{r}\right\}$. Especially, if

$$
k \leq 2 \alpha+2+p \min \left\{\frac{k}{p}+\frac{2 \alpha+1}{p^{\prime}}, \frac{m-1}{r}\right\},
$$

then $f=0$ almost everywhere. Furthermore, if $m \in] 1,1+r]$ and $k>2 \alpha+2$, then $f$ is a constant multiple of $N(., b)$.

iii) If $a b<\frac{1}{4}$, then for all $\left.\delta \in\right] b, \frac{1}{4} a[$ all functions of the form $f(x)=P(x) N(x, \delta)$ satisfy (2.1) and (2.2).

Proof. It follows from $(2.1)$ that $f \in L_{Q}^{1}$ and $\mathcal{F}_{Q}(f)(\xi)$ exists for all $\xi \in \mathbb{R}$. Moreover, it has an entire holomorphic extension on $\mathbb{C}$ satisfying for some $s>0$,

$$
\left|\mathcal{F}_{Q}(f)(z)\right| \leq C e^{\frac{I m z^{2}}{4 a}}(1+|\operatorname{Im} z|)^{s} .
$$

By (1.1) we have for all $z=\xi+i \eta \in \mathbb{C}$,

$$
\begin{aligned}
\left|\mathcal{F}_{Q}(f)(z)\right| & \leq \int_{\mathbb{R}}|f(x)|\left|\Lambda_{\xi}(x)\right||x|^{2 \alpha+1} d x \\
& \leq e^{\frac{\eta^{2}}{4 a}} \int_{\mathbb{R}} \frac{e^{a x^{2}} Q(x)|f(x)|}{(1+|x|)^{\frac{k}{p}}}(1+|x|)^{\frac{k}{p}} e^{-a\left(x-\frac{\eta}{2 a}\right)^{2}}|x|^{2 \alpha+1} d x .
\end{aligned}
$$

By Hölder inequality we have

$$
\left|\mathcal{F}_{Q}(f)(z)\right| \leq e^{\frac{\eta^{2}}{4 a}}\left(\int_{\mathbb{R}} \frac{e^{p a x^{2}} Q(x)^{p}|f(x)|^{p}}{(1+|x|)^{k}}|x|^{2 \alpha+1} d x\right)^{\frac{1}{p}}\left(\int_{\mathbb{R}}(1+|x|)^{\frac{k p^{\prime}}{p}} e^{-a p^{\prime}\left(x-\frac{\eta}{2 a}\right)^{2}}|x|^{2 \alpha+1} d x\right)^{\frac{1}{p^{\prime}}}
$$

according to (2.1) we get that

$$
\begin{aligned}
\left|\mathcal{F}_{Q}(f)(\xi+i \eta)\right| & \leq C e^{\frac{\eta^{2}}{4 a}}\left(\int_{\mathbb{R}}(1+|x|)^{\frac{k p^{\prime}}{p}} e^{-a p^{\prime}\left(x-\frac{\eta}{2 a}\right)^{2}}|x|^{2 \alpha+1} d x\right)^{\frac{1}{p^{\prime}}} \\
& \leq C e^{\frac{\eta^{2}}{4 a}}\left(\int_{0}^{\infty}(1+|x|)^{\frac{k p^{\prime}}{p}+2 \alpha+1} e^{-a p^{\prime}\left(x-\frac{\eta}{2 a}\right)^{2}} d x\right)^{\frac{1}{p^{\prime}}} \\
& \leq C e^{\frac{\eta^{2}}{4 a}}(1+|\eta|)^{\frac{k}{p}+\frac{2 \alpha+1}{p^{\prime}}} .
\end{aligned}
$$

If $a b=\frac{1}{4}$, then

$$
\left|\mathcal{F}_{Q}(f)(\xi+i \eta)\right| \leq C e^{b \eta^{2}}(1+|\eta|)^{\frac{k}{p}+\frac{2 \alpha+1}{p^{\prime}}} .
$$

We put $g(z)=e^{b z^{2}} \mathcal{F}_{Q}(f)(z)$, then

$$
|g(z)| \leq C e^{b|\operatorname{Re} z|^{2}}(1+|\operatorname{Im} z|)^{\frac{k}{p}+\frac{2 \alpha+1}{p^{\prime}}} .
$$

It follows from (2.2) that

$$
\int_{\mathbb{R}} \frac{|g(z)|^{r}}{(1+|\xi|)^{m}} d \xi<\infty
$$


Lemma 2.1. Let $h$ be an entire function on $\mathbb{C}$ such that

$$
|h(z)| \leq C e^{a|\operatorname{Re} z|^{2}}(1+|\operatorname{Im} z|)^{l}
$$

for some $l>0, a>0$ and

$$
\int_{\mathbb{R}} \frac{|h(x)|^{r}}{(1+|x|)^{m}}|P(x)| d x<\infty
$$

for some $r \geq 1, m>1$ and $P$ is a polynomial with degree $m$. Then $h$ is a polynomial with degh $\leq \min \left\{l, \frac{m-M-1}{r}\right\}$ and if $m \leq r+M+1$, then $h$ is a constant.

From this Lemma, $g$ is a polynomial, we say $P_{b}$ with $\operatorname{deg} P_{b} \leq \min \left\{\frac{k p^{\prime}}{p}+\frac{2 \alpha+1}{p^{\prime}}, \frac{m-1}{r}\right\}$. Then $\mathcal{F}_{Q}(f)(x)=P_{b}(x) e^{-b x^{2}}$ then,

$$
f(x)=Q_{b}(x) N(x, b)
$$

where $\operatorname{deg} P_{b}=\operatorname{deg} Q_{b}$. Therefore, nonzero $f$ satisfies (1.10) provided that

$$
k>2 \alpha+2+p \min \left\{\frac{k p^{\prime}}{p}+\frac{2 \alpha+1}{p^{\prime}}, \frac{m-1}{q}\right\} .
$$

If $m<r+1$, by Lemma 1 we have $g$ as a constant and $\mathcal{F}_{Q}(f)(x)=C e^{-b x^{2}}$ and $f(x)=C N(x, b)$. If $m>1$ and $k>2 \alpha+2$, these functions satisfy (2.1) and (2.2), which proves (ii).

If $a b>\frac{1}{4}$, then we can find positive constants $a_{1}$ and $b_{1}$ such that $a>a_{1}=$ $\frac{1}{4 b_{1}}>\frac{1}{4 b}$. Then $f$ and $\mathcal{F}_{Q}(f)$ also satisfy (2.2) with $a$ and $b$ replaced by $a_{1}$ and $b_{1}$ respectively. Then $\mathcal{F}_{Q}(f)(x)=P_{b_{1}}(x) e^{-b_{1} x^{2}} . \mathcal{F}_{Q}(f)$ cannot satisfy (2.2) unless $P_{b_{1}}=0$, which implies that $f=0$, this proves (i). If $a b<\frac{1}{4}$, then for all $\left.\delta \in\right] b, \frac{1}{4 a}[$, the functions of the form $f(x)=P(x) N(x, \delta)$, where $P$ is a polynomial on $\mathbb{R}$, satisfy (2.1) and (2.2). This proves (iii).

\section{Mathematical Formulas}

\section{Miyachi's Theorem for the Q-Fourier-Dunkl Transform}

Theorem 4.1. Let $f$ be a measurable function on $\mathbb{R}$ such that

$$
e^{a x^{2}} f \in L_{Q}^{p}(\mathbb{R})+L_{Q}^{r}(\mathbb{R})
$$

and

$$
\int_{\mathbb{R}} \log ^{+} \frac{\left|\mathcal{F}_{Q}(f)(\xi) e^{b \xi^{2}}\right|}{\lambda} d \xi<\infty
$$

for some constants $a, b, \lambda>0$ and $1 \leq p, r \leq+\infty$. 
(i) if $a b>\frac{1}{4}$ then $f=0$ almost everywhere.

(ii) if $a b=\frac{1}{4}$ then $f=c N(., b)$ with $|c| \leq \lambda$.

(iii) if $a b>\frac{1}{4}$ then for all $\left.\delta \in\right] b, \frac{1}{4}[$, all functions of the form $f(x)=P(x) N(x, \delta)$, where $P$ is a polynomial on $\mathbb{R}$ satisfy (2.1) and (2.2).

To prove this result, we need the following lemmas.

Lemma 4.1. [5] Let $h$ be an entire function on $\mathbb{C}$ such that

$$
|h(z)| \leq A e^{B|\operatorname{Re} z|^{2}}
$$

and

$$
\int_{\mathbb{R}} \log ^{+}|h(y)| d y<\infty,
$$

for some constants $A$ and $B$. Then $h$ is a constant.

Lemma 4.2. Let $r \in[1,+\infty], a>0$. Then for $g \in L_{Q}^{r}(\mathbb{R})$ there exist $c>0$ such that

$$
\left\|e^{a x^{2} t} X_{Q}\left(e^{-a y^{2}} g\right)\right\|_{r} \leq c\|g\|_{r, Q} .
$$

Proof. From the hypothesis, it follows that $e^{-a y^{2}}$ belongs to $L_{Q}^{1}(\mathbb{R})$. Then by Proposition $1.1,{ }^{t} X_{Q}\left(e^{-a y^{2}} g\right)$ is defined almost everywhere on $\mathbb{R}$. Here we consider two cases:

i) If $r \in[1,+\infty[$ then

$$
\begin{aligned}
\left\|e^{a x^{2} t} X_{Q}\left(e^{-a y^{2}} g\right)\right\|_{r}^{r} & \leq \int_{\mathbb{R}} e^{a r x^{2}}\left(\int_{\mathbb{R}} Q(y) e^{-a y^{2}}|g(y)| d \nu_{x}(y)\right)^{r} d x \\
& \leq \int_{\mathbb{R}} e^{a r x^{2}}\left(\int_{\mathbb{R}}|Q(y) g(y)|^{r} d \nu_{x}(y)\right)^{\frac{r}{r}}\left(\int_{\mathbb{R}} e^{-a r^{\prime} y^{2}} d \nu_{x}(y)\right)^{\frac{r}{r^{\prime}}} d x
\end{aligned}
$$

where $r^{\prime}$ is the conjugate exponent for $r$. Since

$$
\int_{\mathbb{R}} e^{-r y^{2}} d \nu_{x}(y)=C e^{-r x^{2}},
$$

for $r>0$ it follows from (4.4) that

$$
\begin{aligned}
\left\|e^{a x^{2}{ }^{t}} X_{Q}\left(e^{-a y^{2}} g\right)\right\|_{r}^{r} & \leq C \int_{\mathbb{R}}{ }^{t} X_{Q}\left(|g|^{r}\right)(x) d x \\
& =C \int_{\mathbb{R}}|g(x)|^{r}|x|^{2 \alpha+1} d x<\infty
\end{aligned}
$$

ii) If $r=\infty$ then it follows from (4.4) that

$$
\begin{aligned}
\left\|e^{a x^{2} t} X_{Q}\left(e^{-a y^{2}} g\right)\right\|_{r} & \leq e^{a x^{2} t} X_{Q}\left(e^{-a y^{2}}\right)(x)\|g\|_{Q, \infty} \\
& =C\|g\|_{Q, \infty}
\end{aligned}
$$


Lemma 4.3. Let $r, p \in[1,+\infty]$ and let $f$ be a measurable function on $\mathbb{R}$ such that

$$
e^{a x^{2}} f \in L_{Q}^{p}(\mathbb{R})+L_{Q}^{r}(\mathbb{R})
$$

for some $a>0$. Then for all $z \in \mathbb{C}$, the integral

$$
\mathcal{F}_{Q}(f)(z)=\int_{\mathbb{R}} f(x) \Lambda_{z, Q}(-x)|x|^{2 \alpha+1} d x
$$

is well defined. $\mathcal{F}_{Q}(f)(z)$ is entire and there exists $C>0$ such that for all $\xi, \eta \in \mathbb{R}$,

$$
\left|\mathcal{F}_{Q}(f)(\xi+i \eta)\right| \leq C e^{\frac{\eta^{2}}{4 a}} .
$$

Proof. From (5) and Hölder's inequality we have the first assertion. For (4.6) using (4.5) we have $f \in L_{Q}^{1}(\mathbb{R})$ and ${ }^{t} X_{Q}(f) \in L^{1}(\mathbb{R})$. for all $\xi, \eta \in \mathbb{R}$,

$$
\begin{aligned}
\mathcal{F}_{Q}(f)(\xi+i \eta)=\int_{\mathbb{R}}{ }^{t} X_{Q}(f)(x) e^{-i x(\xi+i \eta)} d x \\
\left|\mathcal{F}_{Q}(f)(\xi+i \eta)\right| \leq\left. e^{\frac{\eta^{2}}{4 a}} \int_{\mathbb{R}} e^{a x^{2}}\right|^{t} X_{Q}(f)(x) \mid e^{-a x^{2}+x \eta-\frac{\eta^{2}}{4 a}} d x \\
\leq e^{\frac{\eta^{2}}{4 a}} \int_{\mathbb{R}} e^{a x^{2}}\left|{ }^{t} X_{Q}(f)(x)\right| e^{-a\left(x-\frac{\eta}{2 a}\right)^{2}} d x .
\end{aligned}
$$

From (4.5) we can deduce that there exists $u \in L_{Q}^{p}(\mathbb{R})$ and $v \in L_{Q}^{r}(\mathbb{R})$ such that

$$
f(x)=e^{-a x^{2}} u(x)+e^{-a x^{2}} v(x),
$$

by Lemma 4 we have

$$
\left.\int_{\mathbb{R}} e^{a x^{2}}\right|^{t} X_{Q}(f)(x) \mid e^{-a\left(x-\frac{\eta}{2 a}\right)^{2}} d x \leq C\left(\|u\|_{p, Q}+\|v\|_{r, Q}\right)<\infty,
$$

which proves the Lemma.

Proof of Theorem

- If $a b>\frac{1}{4}$. Let $h$ be a function on $\mathbb{C}$ defined by

$$
h(z)=e^{\frac{z^{2}}{4 a}} \mathcal{F}_{Q}(f)(z) .
$$

$h$ is entire function on $\mathbb{C}$, it follows from (4.6) that

$$
\forall \xi \in \mathbb{R}, \forall \eta \in \mathbb{R}|h(\xi+i \eta)| \leq C e^{\frac{\xi^{2}}{4 a}} .
$$


On the other hand, we have

$$
\begin{aligned}
\int_{\mathbb{R}} \log ^{+}|h(y)| d y & =\int_{\mathbb{R}} \log ^{+}\left|e^{\frac{y^{2}}{4 a}} \mathcal{F}_{Q}(f)(y)\right| d y \\
& =\int_{\mathbb{R}} \log ^{+} \frac{\left|e^{b y^{2}} \mathcal{F}_{Q}(f)(y)\right|}{\lambda} \lambda e^{\left(\frac{1}{4 a}-b\right) y^{2}} d y \\
& \leq \int_{\mathbb{R}} \log ^{+} \frac{\left|e^{b y^{2}} \mathcal{F}_{Q}(f)(y)\right|}{\lambda} d y+\int_{\mathbb{R}} \lambda e^{\left(\frac{1}{4 a}-b\right) y^{2}} d y
\end{aligned}
$$

because $\log _{+}(c d) \leq \log _{+}(c)+d$ for all $c, d>0$. Since $a b>\frac{1}{4},(2.2)$ implies that

$$
\int_{\mathbb{R}} \log ^{+}|h(y)| d y<\infty .
$$

A combination of (4.7), (4.8) and Lemma 3 shows that $h$ is a constant and

$$
\mathcal{F}_{Q}(f)(y)=C e^{-\frac{1}{4 a} y^{2}}
$$

Since $a b>\frac{1}{4},(2.2)$ holds whenever $C=0$ and the injectivity of $\mathcal{F}_{Q}$ implies that $f=0$ almost everywhere.

- If $a b=\frac{1}{4}$. We deduce from previous case that $\mathcal{F}_{Q}(f)=C e^{-\frac{\xi^{2}}{4 a}}$. Then $(2.2)$ holds whenever $|C| \leq \lambda$. Hence $f=C N(., b)$ with $|C| \leq \lambda$.

- If $a b<\frac{1}{4}$. If $f$ is a given form, then $\mathcal{F}_{Q}(f)(y)=Q(y) e^{-\delta y^{2}}$ for some $Q$.

In the contintion, we will give an analogue of Hardy's theorem [?] for the Q-FourierDunkl transform.

Theorem 4.2. Hardy Let $N \in \mathbb{N}$. Assume that $f \in L_{Q}^{2}(\mathbb{R})$ is such that

$$
|f(x)| \leq M e^{-\frac{1}{4 a} x^{2}} \text { a.e }, \forall y \in \mathbb{R},\left|\mathcal{F}_{Q}(f)(y)\right| \leq M(1+|y|)^{N} e^{-b y^{2}},
$$

for some constants $a>0, b>0$ and $M>0$. Then,

i) If $a b>\frac{1}{4}$, then $f=0$ a.e.

ii) If $a b=\frac{1}{4}$, then the function $f$ is of the form

$$
f(x)=\sum_{|s| \leq N} a_{s} W_{s}\left(\frac{1}{4 a}, x\right) \text { a.e. }, a_{s} \in \mathbb{C} .
$$

iii) If $a b<\frac{1}{4}$, there are infinitely many nonzero functions of $f$ satisfying the conditions (4.9).

Proof. The first condition of (4.9) implies that $f \in L_{Q}^{1}(\mathbb{R})$. So by Proposition 1.1, the function ${ }^{t} X_{Q}(f)$ is defined almost everywhere. By using the relation (1.13), we deduce that for all $x \in \mathbb{R}$,

$$
\left|{ }^{t} X_{Q}(f)(x)\right| \leq M_{0} e^{-a x^{2}},
$$


where $M_{0}$ is a positive constant. So

$$
\left|{ }^{t} X_{Q}(f)(x)\right| \leq M_{0}(1+|x|)^{N} e^{-a x^{2}},
$$

On the other hand from (1.13) and (4.9) we have for all $x \in \mathbb{R}$,

$$
\left|\mathcal{F}\left({ }^{t} X_{Q}\right)(f)(y)\right| \leq M(1+|y|)^{N} e^{-b|y|^{2}},
$$

The relations (4.10) and (4.11) show that the conditions of Proposition 3.4 of [2], p.36, are satisfied by the function ${ }^{t} X_{Q}(f)$. Thus we get:

i) If $a b>\frac{1}{4},{ }^{t} X_{Q}(f)=0$ a.e. Using (1.13) we deduce

$$
\forall y \in \mathbb{R}, \mathcal{F}_{Q}(f)(y)=\mathcal{F} \circ\left({ }^{t} X_{Q}\right)(f)(y)=0 .
$$

Then by the injectivity of $\mathcal{F}_{Q}$ we have $f=0$ a.e.

ii) If $a b=\frac{1}{4}$, then ${ }^{t} X_{Q}(f)(x)=P(x) e^{-a x^{2}}$, where $P$ is a polynomial of degree lower than $N$. Using this relation and (1.13), we deduce that

$$
\forall x \in \mathbb{R}, \mathcal{F}_{Q}(f)(y)=\mathcal{F} \circ^{t} X_{Q}(f)(y)=\mathcal{F}\left(P(x) e^{-\delta x^{2}}\right)(y) .
$$

but

$$
\forall x \in \mathbb{R}, \mathcal{F}\left(P(x) e^{-\delta x^{2}}\right)(y)=S(y) e^{\frac{-y^{2}}{4 \delta}},
$$

with $S$ a polynomial of degree lower than $N$.

Thus from (1.11), we obtain

$$
\forall x \in \mathbb{R}, \mathcal{F}_{Q}(f)(y)=\mathcal{F}_{Q}\left(\sum_{|s|<\frac{N-1}{2}} a_{s} W_{s}\left(\frac{1}{4 \delta}, .\right)\right)(y) .
$$

The injectivity of the transform $\mathcal{F}_{Q}$ implies

$$
f(x)=\sum_{|s| \leq N} a_{s} W_{s}\left(\frac{1}{4 a}, x\right) \text { a.e. }
$$

iii) If $a b<\frac{1}{4}$, let $\left.t \in\right] a, \frac{1}{4 b}\left[\right.$ and $f(x)=C \frac{e^{-t x^{2}}}{Q(x)}$ for some real constant $C$, these functions satisfy the conditions (4.9).

$\square$ In the next part, we will give an analogue of Morgan's theorem [7] for the QFourier-Dunkl transform.

Theorem 4.3. Morgan Let $1<p<2$ and $r$ be the conjugate exponent of $p$. Assume that $f \in L_{Q}^{2}(\mathbb{R})$ satisfies

(4.12) $\int_{\mathbb{R}} e^{\frac{a^{p}}{p}|x|^{p}}|f(x)||x|^{2 \alpha+1} d x<+\infty$, and $\int_{\mathbb{R}} e^{\frac{b^{r}}{r}|y|^{r}}\left|\mathcal{F}_{Q}(f)(y)\right| d y<+\infty$,

for some constants $a>0, b>0$.

Then if $a b>\left|\cos \left(\frac{p \pi}{2}\right)\right|^{\frac{1}{p}}$, we have $f=0$ a.e. 
Proof. The first condition of (4.12) implies that $f \in L_{Q}^{1}(\mathbb{R})$. So by Proposition 1.1, the function ${ }^{t} X_{Q}(f)$ is defined almost everywhere. By using the relation (4.12) and Proposition 1.1, we deduce that:

$$
\left.\left.\int_{\mathbb{R}}\right|^{t} X_{Q}(f)(x)\left|e^{\frac{a^{p}}{p}|x|^{p}} d x \leq \int_{\mathbb{R}} e^{\frac{a^{p}}{p}|x|^{p}}\right| f(x)|| x\right|^{2 \alpha+1} d x<+\infty .
$$

So

$$
\int_{\mathbb{R}}\left|{ }^{t} X_{Q}(f)(x)\right| e^{\frac{a^{p}}{p}|x|^{p}} d x<+\infty
$$

On the other hand, from (1.13) and (4.12) we have:

$$
\int_{\mathbb{R}} e^{\frac{b^{q}}{q}|y|^{q}}\left|\mathcal{F}_{Q}(f)(y)\right| d y=\int_{\mathbb{R}} e^{\frac{b^{q}}{q}|y|^{q}}\left|\mathcal{F}\left({ }^{t} X_{Q}\right)(f)(y)\right| d y<+\infty .
$$

The relations (4.13) and (4.14) are the conditions of Theorem 1.4, p.26 of [2], which are satisfied by the function ${ }^{t} X_{Q}(f)$. Thus we deduce that if $a b>\left|\cos \left(\frac{p \pi}{2}\right)\right|^{\frac{1}{p}}$ we have ${ }^{t} X_{Q}(f)=0$ a.e. Using the same proof as in the end of Theorem 4 , we have obtained $f(y)=0$. a.e. $y \in \mathbb{R}$.

Acknowledgments The authors are deeply indebted to the reviewers for providing constructive comments and helps in improving the contents of this article.

\section{REF E R E N C E S}

1. E. A. Al Zahrani and M. A. Mourou: The Continuous Wavelet Transform Associated with a Dunkl Type Operator on the Real Line. Advances in Pure Mathematics. 3(2013), 443-450.

2. A. Bonami and B. Demange and P. Jaming: Hermite functions and uncertainty principles for the Fourier and the windowed Fourier transforms. Rev. Mat. Iberoamericana 19 (2002), 22-35.

3. M. G. Cowling and J. F. PrICE: Generalizations of Heisenberg inequality, Lecture Notes in Math. 992. Springer, Berlin (1983), 443-449.

4. G. H. Hardy: A theorem concerning Fourier transform. J. London Math. Soc. 8 (1933), 227-231.

5. H. MejJaoli, and M. Salhi: Uncertainty principles for the Weinstein transform, Czechoslovak Mathematical Journal, 61 (136) (2011), 941-974.

6. A. MiYACHi: A generalization of theorem of Hardy, Harmonic Analysis Seminar held at Izunagaoka, Shizuoka-Ken, Japon (1997), 44-51.

7. G. W. Morgan: A note on Fourier transforms. J. London Math. Soc. 9 (1934), 188-192.

Achak Azzedine

Faculty of Science Aïn Chock

Department of Mathematics and Informatics 
University of Hassan II, Casablanca 20100, Morocco achakachak@hotmail.fr

Radouan Daher

Faculty of Science Aïn Chock

Department of Mathematics and Informatics

University of Hassan II, Casablanca 20100, Morocco

r.daher@fsac.ac.ma

Najat Safouane

Faculty of Science Aïn Chock

Department of Mathematics and Informatics

University of Hassan II, Casablanca 20100, Morocco

safouanenajat@live.fr

El Mehdi Loualid

Faculty of Science Aïn Chock

Department of Mathematics and Informatics

University of Hassan II, Casablanca 20100, Morocco

mehdi.loualid@gmail.com 